ISSN 1014-4874

DOI : http://dx.doi.org/10.4314/rj.v27i1.7

\title{
Air Pollution Tracking using PDEs
}

\author{
Marie Emmanuel Ntigura Habingabwa ${ }^{1}$, \\ Fidèle Ndahayo $^{2}$ and Fredrik Berntsson ${ }^{3}$ \\ ${ }^{1}$ Integrated Polytechnic Regional Center, Box 6579, Kigali, Rwanda \\ ${ }^{2}$ National University of Rwanda, Box 117, Butare, Rwanda \\ ${ }^{3}$ Linköping University, S-581 83, Linköping, Sweden
}

\begin{abstract}
In this paper we use the convection-diffusion equation to model the transport of pollutant material through the atmosphere. Such models have a wide range of applications such as predicting the environmental impact from new polluting industrial plants.

In our study we solve the convection-diffusion equation in a two dimensional setting using the Crank-Nicholson method. For our application it is important that the numerical scheme be positive.

The developed numerical model is used to predict the distribution of pollutant material for a few example scenarios. We also discuss the influence of external model parameters such as diffusion coefficient, drift velocity and chemical activity, on the results.
\end{abstract}

Keywords: Air Pollution, Convection-diffusion Equation, Crank-Nicholson method, Polluted Zones, Positive Finite Difference Scheme.

\section{Introduction}

In this work we propose a mathematical model that can be used to track the movement of pollutant material in the atmosphere. Such models are important tools for planning the industrial development of a country since they can be used to predict the impact of polluting industrial plants on, e.g., important tourist sites.

Our mathematical model is based on the classic convection-diffusion equation; which essentially expresses a mass balance statement for the pollutant material as it is transported through the air [1]. In our model we assume that the concentration $\varphi$ of pollutant material satisfies the following 2D convection - diffusion equation:

$\frac{\partial \varphi}{\partial t}+u \frac{\partial \varphi}{\partial x}+v \frac{\partial \varphi}{\partial y}-\mu\left(\frac{\partial^{2} \varphi}{\partial x^{2}}+\frac{\partial^{2} \varphi}{\partial y^{2}}\right)+\sigma \varphi=f$,

where $(u, v)$ are the components of the wind velocity, or drift- velocity vector, $\sigma \geq 0$ is the pollutant chemical transformation coefficient or fall 
out to the ground, $\mu$ is the diffusion coefficient, and $f$ is a source term that describes where the pollutant material is generated and its power.

Besides the time derivative, the left hand side of equation (1.1) has three distinct parts with different physical interpretation. First we have the convection part which describes the movement of pollutant particles due to wind. The wind speed has to be measured and will depend on both time and location. The second part describes how the pollutant spreads through the air due to diffusion. While local variations in air quality may influence the diffusivity constant it is acceptable for our purposes, and as an initial simplification, to treat the diffusivity as a constant. The last term describes how the pollutant material is removed from the air because of, e.g. chemical transformation, or fallout to the ground $[2,3]$. In a practical simulation the coefficient $\sigma$ has to be estimated based on meteorological data such as rain fall and temperature.

In our code we only track pollution movement in a rectangular domain. Thus we need to specify boundary conditions. Choosing appropriate boundary conditions is very important for the accuracy of the results. In our codes we use zero Dirichlet boundary conditions. This is not realistic but the accuracy of the numerical results can still be guaranteed by choosing example scenarios where the pollutant material never moves close to the boundaries during the simulations. This will be explained further in Section 3. More realistic boundary conditions have been developed, see e.g.[2].

The paper is organized as follows: In Section 2 we discuss the discretization of the equation (1.1) using the Crank-Nicholson method. Also we discuss the positiveness of the resulting finite difference scheme. In Section 3 we present a few test scenarios that will be used for evaluating our proposed method. Also the results from numerical calculations are presented. Finally, in Section 4 we summarize our results and discuss future improvements to our codes.

\section{Discretization}

Generally the two dimensional convection-diffusion equation (1.1) cannot be solved analytically. Instead we have developed a finite difference solver based on the Crank-Nicholson method; which is second order accurate in both time and space, see e.g. $[4,5]$. For our application it is important that the components of the numerical solution $\left\{\varphi\left(x_{i}, y_{j}, t_{k}\right)\right\}$ are positive numbers since they represent the concentration of pollutant material. Thus we need to refine the Crank-Nicholson method slightly by including a positiveness condition [2]. 
The computational domain is the rectangle $\Omega=[0, X] \times[0, Y]$ and we simulate the system during a time period $[0, T]$. The space domain is discretized using a uniform grid of size $M N$. The grid parameters are $h=\frac{X}{M}$ and $k=\frac{Y}{N}$.

We introduce a vector $\phi(t)$ that contains the individual concentrations $\varphi\left(x_{i}, y_{j}, t\right)$ at the grid points. By discretizing the space derivatives in (1.1), using finite differences, we obtain a linear system:

$\phi_{t}+A(t) \phi(t)=F(t), A=\frac{u}{h} A_{1}+\frac{v}{k} A_{2}-\frac{\mu}{h^{2}} A_{3}-\frac{\mu}{k^{2}} A_{4}+\sigma I_{,}$

where $F(t)$ is a vector that holds the source strengths $f\left(x_{i}, y_{j}, t\right)$ at the grid points. $A_{n}, n=1,2,3,4$ are defined square $M \times N$ dimensional matrices and $I$ is the identity matrix. Note that generally model parameters will not be constant and if, for instance, the wind speed $u=u(x, y, t)$ then the matrix $u A_{1}$ is replaced by $A_{1}=A_{1}(u, t)$.

In our numerical code the Crank-Nicholson method is used to discretize the time derivative. At each step of the method we need to solve a linear system of equations,

$\left(I-\frac{\tau}{2} A\left(t_{j}\right)\right) \phi\left(t_{j}\right)=\left(I+\frac{\tau}{2} A\left(t_{j-1}\right)\right) \phi\left(t_{j-1}\right)+\frac{\tau}{2}\left(F\left(t_{j}\right)+F\left(t_{j-1}\right)\right)$.

Solving the linear systems represents a significant amount of computational work and a sparse solver is necessary.

Recall that, for our application, we require that the computed concentrations $\phi\left(t_{j}\right)$ are positive [7]. We give the following definitions:

Definition 2.1 A difference scheme is called positive if its solution is nonnegative when its right hand side, initial condition and boundary conditions are all non-negative.

Definition 2.2 A difference scheme is called monotone if it preserves the monotonicity along lines in the space domain between time steps.

It is desirable that our numerical solution is positive and that monotonicity is preserved. This holds if the grid parameters $h, k$ and $\tau$ satisfy the relations $[6,8]$ :

$k<\frac{2 \mu}{v}, h<\frac{2 \mu}{u}, \tau \leq \frac{1}{2 \sigma}$ and $\frac{2-\tau \sigma}{2 \tau \mu}>\frac{1}{k^{2}}+\frac{1}{h^{2}}$

These additional conditions provide information about the optimal grid size parameters $M, N$ in (2.2).

The above finite difference scheme has been implemented using Matlab as a function:

function $[\mathrm{Phi}]=$ Pollution2D(N,M,P,X,Y,T,[u,v],mu,sigma,f,Phi0); 
where $[u, v]$, is a vector (or vector valued function) representing the windspeed, and $m u$,sigma and $f$, are Matlab functions implementing the coefficients in (1.1).

\section{Numerical experiments}

In this section, scenarios which show the influence of the coefficients in the equation (1.1) are presented. In particular we will show the influence of the diffusion coefficient and the drift velocity on the time evolution of polluted zones. The physical interpretation of the results will be discussed. For the simulations we used a uniform grid of size $(N, M)=(80,80)$ and performed $L=80$ time steps. The same transformation coefficient $\sigma=$ 0.05 was used in all tests.

\subsection{The Influence of the Pollutant Diffusion on the Evolution of Polluted Zones}

In the first scenario we assume a single pollutant source of constant strength $f=5$ located in the area $0.4 \leq x \leq 0.6$ and $0.4 \leq y \leq 0.6$. The wind speed is assumed constant $(u, v)=(2,2)$, and thus the pollutant material should mainly spread into the interior of the computational domain. This has the effect of ensuring that the boundary condition $\varphi=0$ is accurate during the simulation. The numerical results are shown in Figure 1.
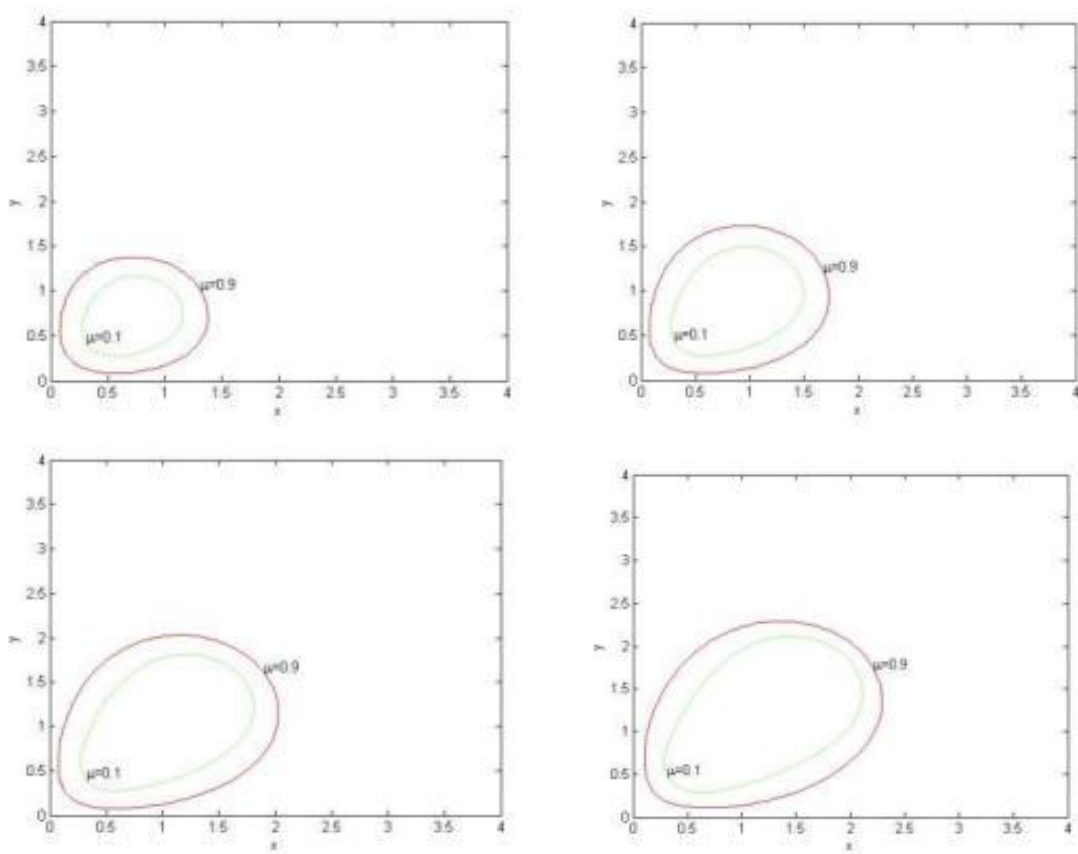

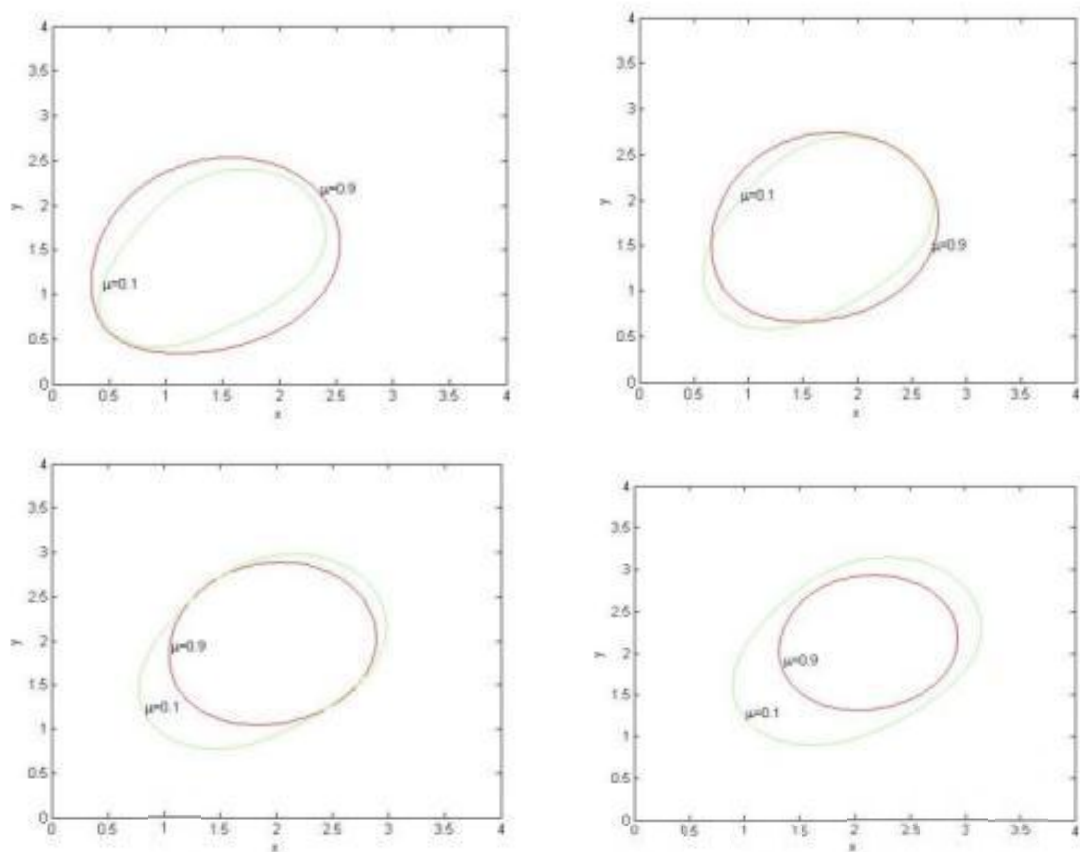

Figure 1: The evolution of the pollutant zones for diffusion coefficient $\mu=0.1$ (green) and $\mu=0.9$ (red). The graphs shows the level curves $\varphi(x, y) \geq 0.01$. The polluted zones are displayed at different datest $=0.7, t=1.2, t=1.7, t=2.2, t=$ $2.7, t=3.2, t=3.7, t=4$.

The diffusion coefficient plays a big role in the evolution of the polluted zones. For a light pollutant, i.e. a high diffusion coefficient, the polluted zone expands rapidly, but also dissipates quickly when the polluting activity is stopped. A heavy pollutant, having a low diffusion constant, only expands slowly, but it also stays longer in polluted regions.

\subsection{The Influence of Drift Velocity on the Evolution of Polluted Zones}

In the second scenario we also assume a single pollutant source of constant strength $f=5$ located in the area $0.4 \leq x \leq 0.6$ and $0.4 \leq y \leq 0.6$. We compare two situations characterized by different constant values of the wind speed $(u, v)=(2,2)$ and $(u, v)=(6,6)$ and thus the pollutant material should mainly spread into the interior of the computational domain. This has the effect of ensuring that the boundary condition $\varphi=0$ is accurate during the simulation. The numerical results are shown in Figure 2. 

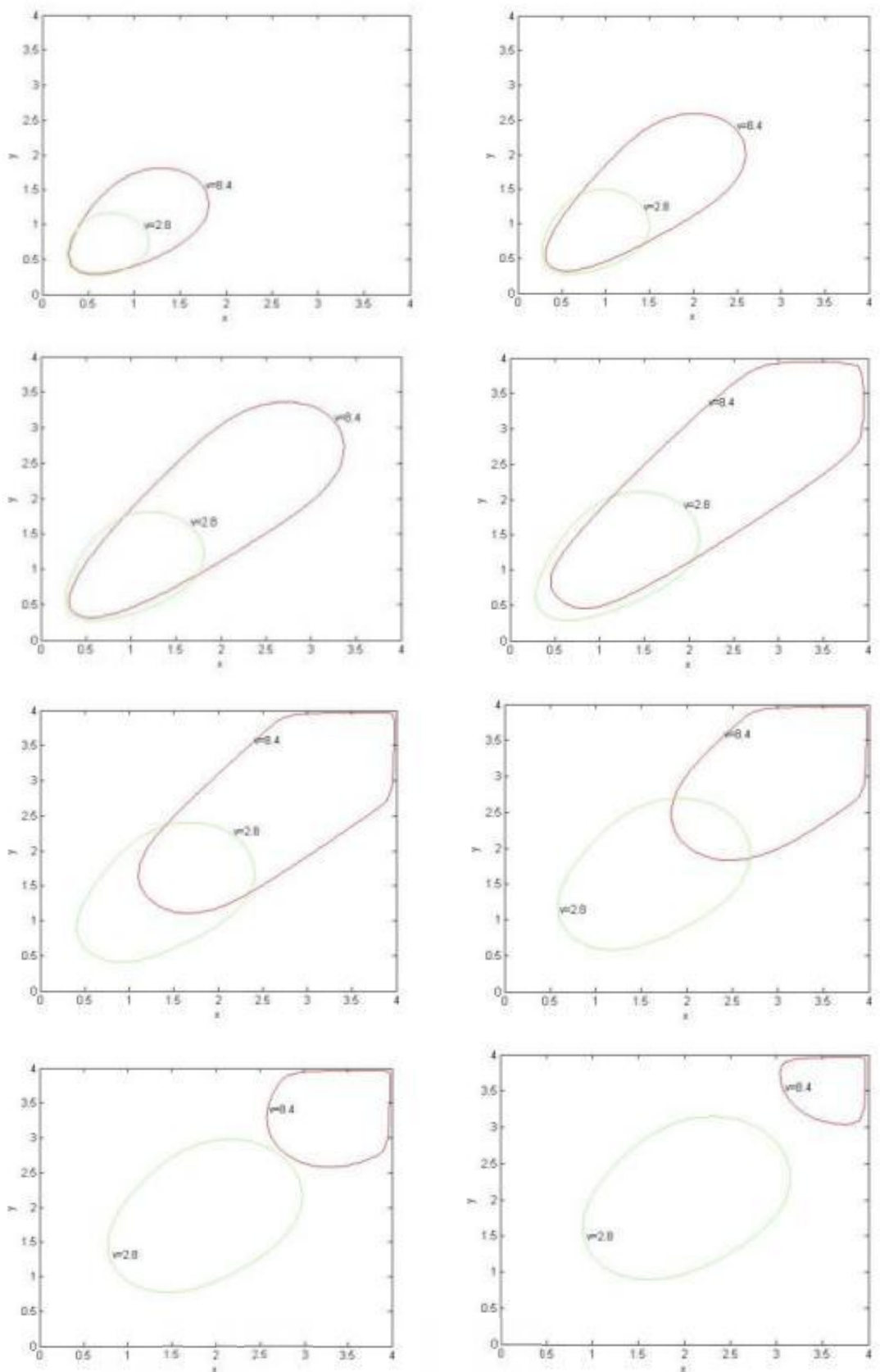

Figure 2: The evolution of polluted zones in two environments with two different drift velocities $v=2.8$ (green) and $v=8.4$ (red). The graphs shows the level curves $\varphi(x, y) \geq 0.01$. The polluted zones are displayed at different dates $t=0.7, t=1.2, t=1.7, t=2.2, t=2.7, t=3.2, t=3.7, t=4$. 
The drift velocity plays a significant role in the expansion of pollutant in the affected region. For a high drift velocity the pollutant spreads on a big region but also quits the observed zone more quickly when the polluting source is stopped. For a low drift velocity it spreads slowly than the previous one and it stays in affected region relatively longer.

\section{Concluding Remarks}

In this paper we discussed the behavior of air pollution using the convection-diffusion equation. We constructed an adequate numerical scheme which is positive and monotone. The graphical representation showed how the pollution concentration changes according to the variation of the parameters equation and the time evolution of the polluted zones.

By this model it is possible to predict the movement of the air pollution and then be able to plan according to data taken on the ground. It will enable us to put industrial plant in a way they will not harm a lot of the population or the environment.

Future work will concentrate on using an adequate boundary condition as the transparent boundary condition in two dimensions, on resolving a full three dimension convection-diffusion equation or a collection of two dimension convection-diffusion equation with different layers where we can include some layers coupling.

\section{References}

[1] Hillel Rubin and Joseph Atkinson Environmental Fluid Mechanics, Marcel Dekker, Inc.New York Basel, 2001.

[2] Dang Quang and Matthias Ehrhardt, Adequate Numerical Solution of Air Pollution Problems by positive Difference Schemes on unbounded Domains, 2006.

[3] Frank R. Spellman and Nancy E. Whiting, Environmental engineer's mathematics handbook, CRC Press, ISBN 1-56670-681-5, 2004

[4] Micheal T. Heath, Scientific Computing: An Introductory Survey, second edtion, The McGraw-Hill, 2005

[5] Richard L. Burden and J. Douglas Faires, Numerical Analysis, 4ed, 1988 [6] A.A. Samarskii, The theory of difference schemes, Dekker, New York, 2001.

[7] V.M. Paskonov, V.I. Polezhaev and L.A. Chudov, Numerical modelling of heat and mass transfer, Moscow, Nauka, 1984

[8] K.O. Friedrichs, Symmetric hyperbolic linear differential equations, Comm.Pure Appl. Math. 7 (1954) 